\title{
Remaining Useful Life Prediction of Power Lithium-Ion Battery based on Artificial Neural Network Model
}

\author{
Enguang Hou ${ }^{a}$, Xin Qiao ${ }^{b}$ and Guangmin Liu ${ }^{c}$
}

China Shandong Provincial Key Laboratory of Automotive Electronic Technology, Institute of Automation, Shandong Academy of Sciences, Jinan 250014 China

ahngauto@163.com, bbugqiao@gmail.com, cliugm@keylab.net

Keywords: Power Lithium-Ion Battery, Remaining Useful Life, Artificial Neural Network Model, Prediction Method.

\begin{abstract}
In order to improve the security and reliability of the power lithium batteries, this paper introduced forecast and health management technology of its core content-remaining useful life, established a power lithium battery remaining useful life prediction method, by collecting current, batteries, battery voltage, temperature, battery SOC and SOH etc data, artificial intelligence model based on neural network, training model parameters, the prediction power lithium battery remaining useful life, simulation results show the advances and reliability of this method.
\end{abstract}

\section{Introduction}

In order to improve the security and reliability of the new energy vehicles, for power lithium battery, we can't just on the battery tube system monitoring function, must be "early detection, early prevention" safety accident. Then, we introduce a concept - forecast and Health Management technology [1] (Prognostics and Health Management, PHM). Forecasting is the core content and the technical challenges of PHM, predict the estimate and predict the Remaining Useful Life of a device or system (RUL, Remaining Useful Life).Residual life prediction for system equipment maintenance is indispensable important information, according to the RUL to predict the results of analysis to good management system, can improve the system or equipment availability and reliability, and reduce or avoid failure caused by heavy losses.

Power Remaining Useful Life of the lithium battery also called power lithium battery residual service life and legacy of life, refers to the system degradation to unacceptable levels before also can use of time. Literature [2] [3] [4] research on lithium ion battery residual life prediction methods are analyzed, summing up the residual life prediction method of the lithium ion battery research present situation, mainly including based on the model, and data fusion model driven prediction method, as shown in figure 1 .

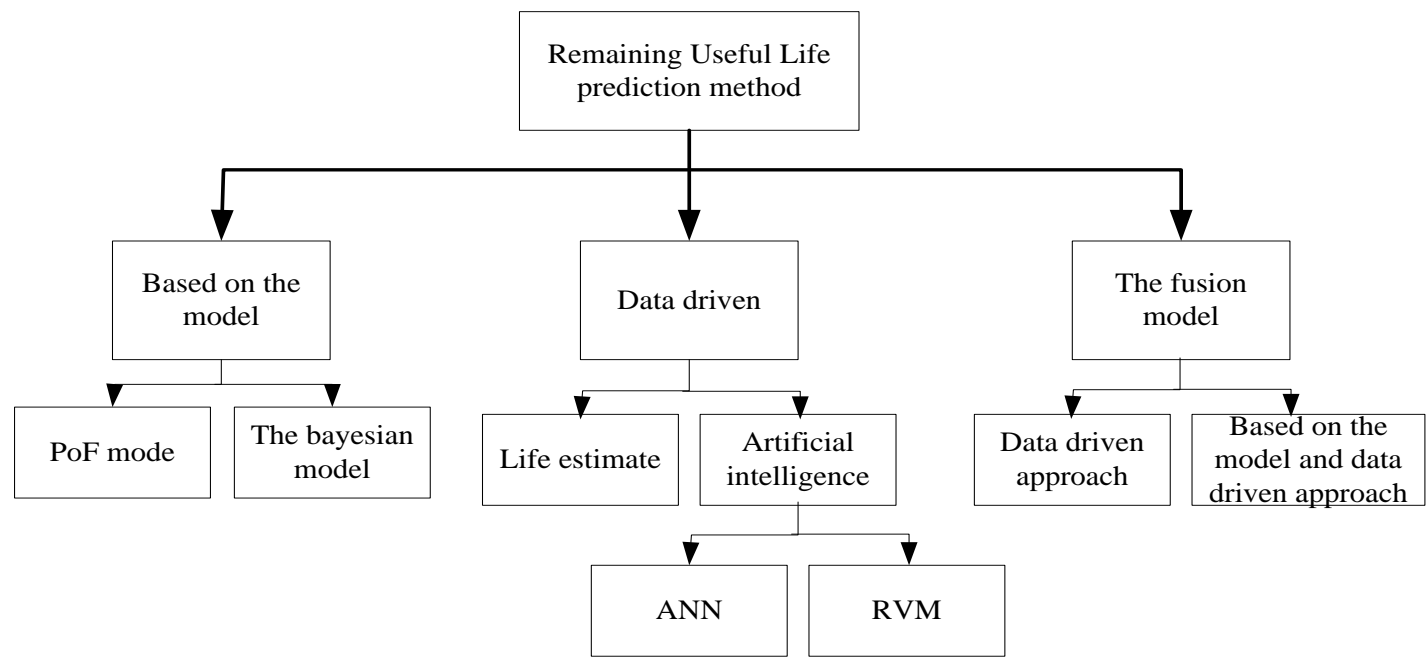

Fig. 1 Remaining Useful Life prediction method 
In this paper, the concept of the remaining useful life (RUL), introduce power lithium battery field, by predicting Remaining Useful Life of the power lithium battery, can be used to predict the security of the power lithium battery failure, make electric cars has higher reliability and security.

\section{Remaining useful life prediction of power lithium-ion battery}

\subsection{The influence factors.}

Power lithium battery remaining service life is the main influence factors including total voltage, temperature, charge and discharge current, SOC, batteries voltage, $\mathrm{SOH}$ and so on six big factors.

(1) The total voltage, expressed as $\mathbf{X}_{1}$ the range of total voltage from $285 \mathrm{~V}$ to $390 \mathrm{~V}$, the battery pack is 95 series of lithium battery, the total voltage of representing the depth of the battery pack charging and discharging;

(2) Temperature, expressed as $\mathbf{X}_{2}$ : temperature ranges from $-35{ }^{\circ} \mathrm{C}$ to $75{ }^{\circ} \mathrm{C}$, the battery pack has a temperature of 24 points, to select the temperature of the battery pack with the T12 representative.

(3) The charge and discharge current, expressed as the $\mathbf{X}_{\mathbf{3}}$ : current range - $0.1-\mathrm{C}$ to $3 \mathrm{C}$, negative charging, discharge is positive.

(4) SOC of the battery pack, expressed as $\mathbf{X}_{\mathbf{4}}$ : choose to represent most of the single section SOC of batteries, this article selects a list of 10 .

(5) The batteries voltage, expressed as $\mathbf{X}_{5}$ : choose to represent most of the single section batteries voltage, this paper selected a list of 10 , consistent with the selection of SOC;

(6) $\mathrm{SOH}$ of the battery, represented by $\mathbf{X}_{6}$ : select 10 strings, agree with SOC, batteries voltage selection, this article selects the 50 and 2.2 Ah battery, the battery pack for the capacity of $110 \mathrm{Ah}$.

\subsection{Power lithium battery remaining useful life of quantification.}

Power lithium battery remaining service life, identified as $\mathrm{Y}_{1}$, this article selects the service life of the cell cycle theory for an 800 - cycle, each charge and discharge decreases.

$\mathrm{Y}_{1}=(800-$ cycle $) / 800$.

\section{The data collection}

In this paper, the data of real vehicle running data, according to the above principles, through post screening form, part of the data in table 1 .

Table 1. The data collection

\begin{tabular}{|c|c|c|c|c|c|c|c|c|}
\hline Time & $\mathrm{NO}$ & $\mathrm{X}_{1} / \mathrm{V}$ & $\mathrm{X}_{2} /{ }^{\circ} \mathrm{C}$ & $\mathrm{X}_{3} / \mathrm{A}$ & $\mathrm{X}_{4} /$ & $\mathrm{X}_{5} / \mathrm{V}$ & $\mathrm{X}_{6} / \mathrm{Ah}$ & $\mathrm{Y}_{1} /$ \\
\hline 2015.12 .1 & 1 & 354.89 & 20 & 0.00 & $49.2 \%$ & 3.733 & 110 & $100.0 \%$ \\
\hline 2015.12 .1 & 2 & 353.46 & 20 & 1.78 & $47.6 \%$ & 3.726 & 110 & $100.0 \%$ \\
\hline 2015.12 .1 & 3 & 345.285 & 21 & 19.91 & $44.2 \%$ & 3.64 & 110 & $100.0 \%$ \\
\hline 2015.12 .1 & 4 & 347.475 & 21 & 2.76 & $44.1 \%$ & 3.663 & 110 & $100.0 \%$ \\
\hline 2015.12 .1 & 5 & 356.373 & 15 & -8.54 & $56.7 \%$ & 3.753 & 110 & $100.0 \%$ \\
\hline 2015.12 .1 & 6 & 366.401 & 16 & -8.54 & $71.7 \%$ & 3.859 & 110 & $99.9 \%$ \\
\hline 2015.12 .1 & 7 & 367.661 & 16 & -8.54 & $73.5 \%$ & 3.871 & 109 & $99.9 \%$ \\
\hline 2015.12 .1 & 8 & 369.331 & 17 & -8.48 & $75.9 \%$ & 3.889 & 109 & $99.9 \%$ \\
\hline \multicolumn{8}{|c|}{ Limited space, 774 groups of data from here } & \\
\hline 2016.4 .5 & 783 & 350.32 & 46 & 32.74 & $40.1 \%$ & 3.705 & 103 & $90.3 \%$ \\
\hline 2016.4 .5 & 784 & 342.929 & 44 & -18.96 & $47.7 \%$ & 3.642 & 103 & $90.3 \%$ \\
\hline
\end{tabular}

\section{Based on neural network model is established in this paper}

Structure diagram as shown in figure 2, this paper USES the artificial neural network of artificial neural network can learn and store a lot of input - output model mapping, without prior mathematical equation describing the mapping relation [5]. 


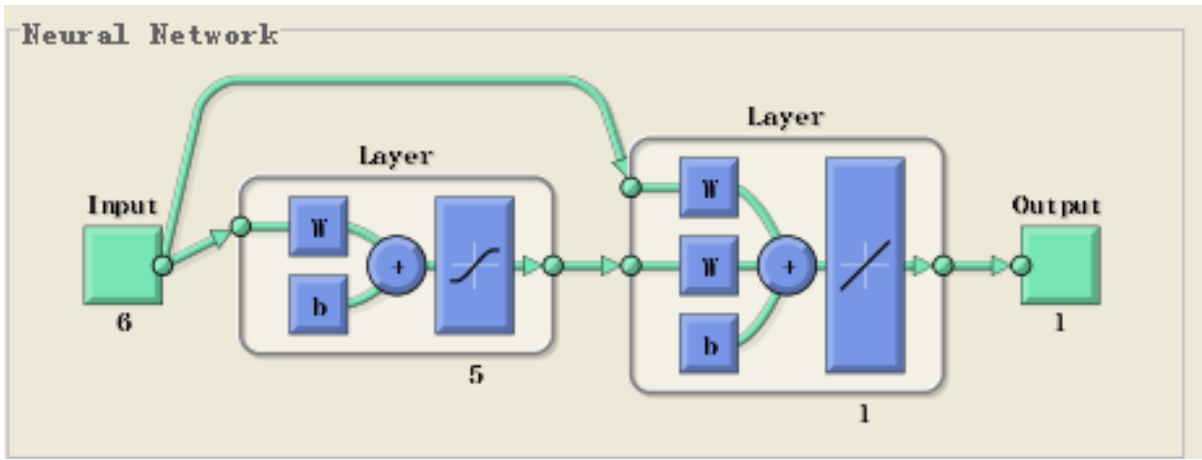

Fig. 2 Neural network structure

The establishment of the artificial neural network:

(1)Input $=\left\{\mathrm{X}_{1}, \mathrm{X}_{2}, \mathrm{X}_{3}, \mathrm{X}_{4}, \mathrm{X}_{5}, \mathrm{X}_{6}\right\}$.

(2)Output $=Y_{1}$.

(3)Network Layer: to establish a neural network, the first thing to determine in advance of network layer, without limiting the number of hidden layer nodes, two layer (only one hidden layer) can achieve arbitrary nonlinear mapping of neural network.

(4)Determine the number of nodes in the hidden layer: For pattern recognition of neural network, the number of nodes in the hidden layer design with reference to the following formula.

$$
n=\sqrt{n_{i}+n_{0}}+a
$$

Where, the $\mathrm{n}$ is as the number of nodes in the hidden layer. The $\mathrm{n}_{\mathrm{i}}$ The input node number.

The $\mathrm{n}_{0}$ is the output node number. The a is $1 \sim 10$ between constant. This article selects the number of hidden layer is 5 .

(5) Transfer function: according to the need to choose the $S$ type (sigmoid) function, such as pure linear function.

(6) Training function: through a variety of training function, optimize data, the purpose of training.

According to the charge and discharge cycle to the input values and the corresponding output values, the parameters of the neural network training, the training set is reached after parameter, to stop training, the training parameters. The parameters in the process of artificial neural network training diagram, as shown in figure 3.

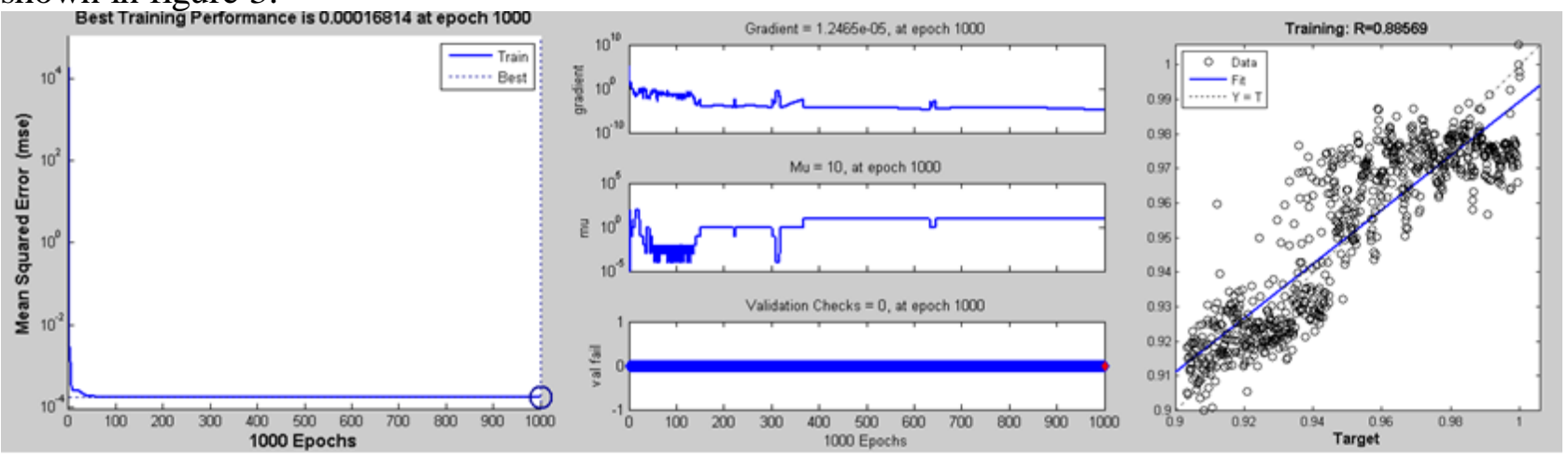

Fig. 3 Performance, training state and Regression

\section{Experimental verification}

Run by real vehicle road, 10 groups of data, using new collection of 10 sets of data, as a test input variables, verification, the results are shown in table 2 .

As can be seen from table 2, the influence factors such as input voltage and current data, test results, compared with the data collected, within $\pm 5 \%$ error rate basically can control, so as to realize the total voltage, temperature, charge and discharge current through gathering, SOC, batteries voltage, SOH six elements such as data, prediction power lithium battery remaining service life of purpose, in order to improve the safety and reliability of the power lithium battery to provide the basis. 
Table 2. The simulation verification table

\begin{tabular}{|c|c|c|c|c|c|c|c|c|c|c|}
\hline Time & $\mathrm{NO}$ & $\mathrm{X}_{1} / \mathrm{V}$ & $\mathrm{X}_{2} /{ }^{\circ} \mathrm{C}$ & $\mathrm{X}_{3} / \mathrm{A}$ & $\mathrm{X}_{4} /$ & $\mathrm{X}_{5} / \mathrm{V}$ & $\mathrm{X}_{6} / \mathrm{Ah}$ & $\begin{array}{c}\text { Real } \\
\mathrm{Y}_{1} /\end{array}$ & $\begin{array}{c}\mathrm{Test} \\
\mathrm{Y}_{\mathrm{C}} /\end{array}$ & error \\
\hline 2016.5 .1 & 1 & 360.16 & 19 & 40.22 & $75.2 \%$ & 3.772 & 103 & $90.3 \%$ & $88.0 \%$ & $2.4 \%$ \\
\hline 2016.5 .1 & 2 & 356.14 & 19 & 68.60 & $71.5 \%$ & 3.718 & 103 & $90.3 \%$ & $91.9 \%$ & $-1.6 \%$ \\
\hline 2016.5 .1 & 3 & 356.82 & 20 & 29.70 & $66.7 \%$ & 3.73 & 103 & $90.3 \%$ & $91.5 \%$ & $-1.2 \%$ \\
\hline 2016.5 .1 & 4 & 336.40 & 20 & 121.53 & $59.5 \%$ & 3.519 & 103 & $90.3 \%$ & $91.9 \%$ & $-1.6 \%$ \\
\hline 2016.5 .1 & 5 & 351.38 & 22 & -16.76 & $57.3 \%$ & 3.681 & 103 & $90.3 \%$ & $85.8 \%$ & $4.5 \%$ \\
\hline 2016.5 .1 & 6 & 347.97 & 22 & -15.92 & $52.3 \%$ & 3.646 & 103 & $90.3 \%$ & $93.5 \%$ & $-3.2 \%$ \\
\hline 2016.5 .1 & 7 & 345.43 & 22 & -18.92 & $47.8 \%$ & 3.621 & 103 & $90.3 \%$ & $91.4 \%$ & $-1.1 \%$ \\
\hline 2016.5 .1 & 8 & 338.69 & 22 & 15.77 & $39.4 \%$ & 3.549 & 102 & $90.2 \%$ & $87.8 \%$ & $2.4 \%$ \\
\hline 2016.5 .1 & 9 & 339.49 & 23 & -17.75 & $37.6 \%$ & 3.552 & 102 & $90.2 \%$ & $91.4 \%$ & $-1.2 \%$ \\
\hline 2016.5 .1 & 10 & 328.57 & 23 & 33.63 & $27.9 \%$ & 3.436 & 102 & $90.2 \%$ & $89.4 \%$ & $0.8 \%$ \\
\hline
\end{tabular}

\section{Conclusion}

This paper introduced the concept of the remaining useful life (RUL), through the real vehicle road test, gathering effect power lithium battery residual service life of the influence factors of the data, set up power lithium battery remaining service life of artificial neural network model, and parameters of the training, establish power lithium battery remaining service life prediction method.

The reliability of the test results show that the artificial neural network model, the error in the range of the ideal, illustrates the advanced prediction method.

\section{Acknowledgments}

This paper is supported by Shandong Provincial Natural Science Foundation, China (ZR2015 FQ007 and ZR2014HM031), and focus on research and development plan in Shandong province (major key technologies) (2016ZDJS03A04).

\section{References}

[1]. Sikorska J.Z., Ma L. Prognostic modeling options for remaining useful life estimation by industry [J]. Mechanical Systems and Signal Processing, 2011, 25(5):1803-1836.

[2]. Michael P., Rubyca J. A prognostics and health management roadmap for information and electronics-rich systems [J].Microelectronics Reliability, 2010, 50(3):317-323.

[3]. LUO Wei-lin, ZHANG Li-qiang, LV Chao, WANG Li-xin. Review on Foreign Status of Life Prediction of Lithium-ion Batteries [J]. Journal of Power Supply. 2013(1):140-144.

[4]. Xu Jiali. Study on Fault Prognostic and Health Management for Electronic System [D].ChengDu. University of Electronic Science and Technology of China. 2009:1-11

[5]. HOU En-guang1, LI Shu-bin1, LI Ke1. Neural network based assessment of pharmacological effects of traditional Chinese medicine [J]. ,Shandong Science, 2011,24(1):72-76. 